\title{
Redescription of Lycidas chrysomelas (Simon) (Araneae: Salticidae)
}

\author{
Julianne M. Waldock \\ Western Australian Museum, Francis Street, Perth, \\ Western Australia 6000, Australia
}

\begin{abstract}
Lycidas chrysomelas, peviously known from only the holotype collected from Lion Mill, Western Australia, is found to be widespread in semi-arid Australia. The female is described for the first time, and the male is redescribed.
\end{abstract}

\section{INTRODUCTION}

In 1909 Eugene Simon described Habrocestum chrysomelas based on a single male that had been collected by Michaelsen and Hartmeyer on their expedition to south-western Australia in 1905. Subsequent published accounts have been limited to catalogue listings (Rainbow, 1911; Roewer, 1954; Bonnet, 1957) and a description of the male holotype and only known specimen by Żabka (1987). Recent collections of spiders from different regions of Australia have included many specimens of L. chrysomelas, which indicate that the spider has a widespread distribution in drier, semi-arid regions of the continent. I here present a redescription of the male based on recent material and the first description of the female of this little-known species.

\section{MATERIALS AND METHODS}

Material is lodged in the Western Australian Museum (WAM), South Australian Museum (SAM), Museum of Victoria (NMV), and Zoologisches Museum der Humboldt-Universität (ZMB). The names of some collectors have been abbreviated as follows in the Material Examined section: M.S. Harvey (MSH), J.M. Waldock (JMW), A.F. Longbottom (AFL), G. Friend (GF), D. Hirst (DH), P. Van Heurck (PVH), N.A. Guthrie (NG), B. Durrant (BD), L. King (LK), and material from the Salinity Action Plan Survey (SAP).

The female genitalia were examined by dissecting the epigynum from the abdomen and clearing it in $10 \%$ lactic acid for 10 minutes in a warm water bath. The epigynum was mounted in glycerol and drawn with a camera lucida fitted to an Olympus compound microscope. Other drawings were made using a graticule fitted to a Leica dissecting microscope.

\section{SYSTEMATICS}

Family Salticidae

Genus Lycidas Karsch

Lycidas Karsch, 1878: 25; Żabka, 1987: 451.

Jotus L. Koch, 1881: 1243. Synonymized by Żabka, 1987: 451.

\section{Type species}

of Lycidas: Lycidas anomalus Karsch, 1878: 26, by monotypy.

of Jotus: Jotus auripes L. Koch, 1881: 1243, by subsequent designation of Simon, 1901: 566.

\section{Remarks}

The male palpal structures indicate that the genera Lycidas and Maratus Karsch have a close affinity as Żabka (1987) has indicated. Features of the female genitalia and the general body configurations distinguish these salticids as separate genera. The presence of modified scutes and squamous hairs in males of both genera also indicate affinities, however the modifications of the scutes distinguish the genera. Males of Maratus have the abdominal scute developed into lateral flaps that fold under the abdomen, the scute is covered in squamous hairs of various colours and patterns. In species of Lycidas related to $L$. chrysomelas the males also have a scute with coloured squamous hairs but the scute is not developed into lateral flaps and there is a fringe of hairs along the edges. The tip of the embolus appears to be associated very closely with a conductor in Lycidas while in Maratus the conductor is more separated. The female genitalia of both Lycidas and Maratus are similar in that the spermatheca tend to be large but $L$. chrysomelas and related species have large, copulatory canals and 
other structures such as proximal receivers are not evident. Proximal receivers are present in Maratus species.

\section{Lycidas chrysomelas (Simon, 1909) Figures $1-10$}

Habrocestum chrysomelas Simon, 1909: 201; Rainbow, 1911: 296; Roewer, 1954: 1119; Bonnet, 1957: 2064.

Lycidas chrysomelas (Simon): Żabka, 1987: 457, figures 19-22.

\section{Material Examined}

\section{Holotype}

ð, Lion Mill [= Mt Helena], Western Australia, Australia [31 $\left.53^{\prime} \mathrm{S}, 116^{\circ} 12^{\prime} \mathrm{E}\right]$, stat. 99, 22 May - 11 October 1905, W. Michaelsen, R. Hartmeyer (ZMB 18603).

\section{Other Material}

Australia: New South Wales: 2 o, Finlay [Finley], $35^{\circ} 39^{\prime} \mathrm{S}, 145^{\circ} 34^{\prime} \mathrm{E}, 16$ February 1934, W.B. White (NMV). South Australia: $1 \delta, 1 \mathrm{~km}$ E. of Anamba East Dam, pitfall trap, 32 $47^{\prime} 00^{\prime \prime} \mathrm{S}, 140^{\circ} 15^{\prime} 30^{\prime \prime} \mathrm{E}, 5-9$ October 1992, N. P. W. S. South Olary Plain Survey (SAM N199578); 1 \%, $2.5 \mathrm{~km}$ NW. of Bull Dam, pitfall, 32³1'30"S, $140^{\circ} 49^{\prime} 10^{\prime \prime} \mathrm{E}, 28$ September - 2 October 1992, N. P. W. S. South Olary Plain Survey (SAM N199579); $1 \delta, 2.5 \mathrm{~km}$ NNE. of Glenora, 3327'30"S, 139²2'00"E, 23 October 1992, N. P. W. S. South Olary Plain Survey (SAM N199581); 1 , Granite Downs [Station], near camp, 265ㅇ' 13330'E, December 1984, B. Guerin (SAM N1999501); 1 đ, Hallett Cove, pitfall, 3505'S, 138 $30^{\circ}$ E, 25 September 1994, A.J. McArthur (SAM N1997916); 1 \%, $2 \mathrm{~km} \mathrm{~W}$. of Hanging Knoll, pitfall, $26^{\circ} 19^{\prime 2} 23^{\prime \prime S}, 130^{\circ} 23^{\prime} 36^{\prime \prime} \mathrm{E}, 4-8$ May 1993, Pitjantjatjara Lands Survey, WW0601 (SAM N199589); 1 \%, 1 \%, Katarapko Game Reserve (= Murray River National

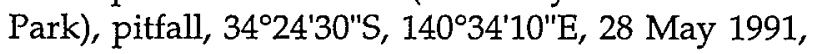
A.J. McArthur (SAM N199583, N1995585); 1 б, 13 $\mathrm{km} \mathrm{N}$. of Keilira, $36^{\circ} 35^{\prime} \mathrm{S}, 140^{\circ} 10^{\prime} \mathrm{E}, 7$ May $1975, \mathrm{DH}$

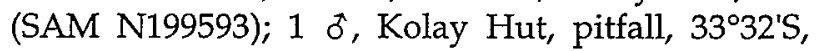
135³6'E, 8-11 December 1989, DH (SAM N199587); 1 ๙, $17.5 \mathrm{~km}$ ENE. of Mt Cooperinna, pitfall, 2619'20"S, 13007'56"E, 6 May 1994, Pitjantjatjara Lands Biological Survey (SAM N199590); 1 ð, 7.5 $\mathrm{km}$ NNW. of Mt Kintore, pitfall, 26 29'56"S, 130²7'22"E, 9 May 1994, Pitjantjatjara Lands Biological Survey, WA0401 (SAM N199591); $1 \delta$, Muckera Rockhole, pitfall, $30^{\circ} 02^{\prime} \mathrm{S}, 130^{\circ} 03^{\prime} \mathrm{E}$, September 1984, B. Guerin (SAM N1999496); 1 o',

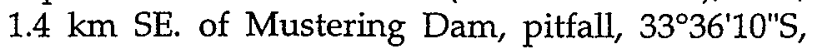
$139^{\circ} 37^{\prime} 40^{\prime \prime}$ E, 28 September - 31 October 1992, N. P. W. S. South Olary Plain Survey, KM0201 (SAM N199580); 1 o, Nangkita, burnt plot 6 Brawley's
Property, $35^{\circ} 22^{\prime} \mathrm{S}, 138^{\circ} 44^{\prime} \mathrm{E}, 30$ January - 3 February 1998 , S. Roberts (SAM N1999492); $1 \delta, 9 \mathrm{~km} \mathrm{SE}$. of Old Boolcoomata, pitfall, $32^{\circ} 10^{\prime} 36^{\prime \prime S}, 140^{\circ} 18^{\prime} 04^{\prime \prime} \mathrm{E}$, 26-31 August 1996, North Olary Plains Survey, OLA056 (SAM N1997914); 1 \%, Poochera, Mudges Heritage site, pitfall, $32^{\circ} 43^{\prime} \mathrm{S}, 134^{\circ} 50^{\prime} \mathrm{E}, 29$ October 7 November 1995, A.J. McArthur, Nothomyrmecia Search (SAM N1997920); 10 , same data except 7 November - 12 December 1995 (SAM N1997919); 1 $\delta, 5 \mathrm{~km}$ S. of Quinyambie, pitfall, $30^{\circ} 15^{\prime} 28^{\prime \prime} \mathrm{S}$, 1405'11"E, 1-6 October 1996, North Olary Plains Survey, CNE091 (SAM N1997915); 1 \%, Salisbury North, [344ㄴ' $138^{\circ} 37^{\prime} \mathrm{E}$ ], 11 April 1982, DH (SAM N199592); 1 \%, $4.4 \mathrm{~km} \mathrm{SE}$. of Sheep Camp Waterhole, pitfall, $29^{\circ} 45^{\prime} 41^{\prime \prime} \mathrm{S}, 136^{\circ} 57^{\prime} 10^{\prime \prime} \mathrm{E}, 30$ September 1995, D.E.L.M. [Department for Environment and Land Management], STO4 (SAM N1997917); 1 ठ, Snodgrass Farm, $22.5 \mathrm{~km}$. SW. of Loxton, pitfalls, 34³3'S, 140²2'E, 28 May 1991, A.J. McArthur (SAM N199582); $10,10.5 \mathrm{~km} \mathrm{SE}$. of

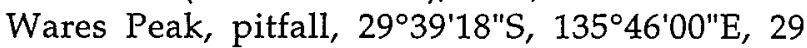
September - 5 October 1995, H.M. Owens, Painted Hills Survey (SAM N1997918); 1 o, $500 \mathrm{~m}$ SW. of Wataru, base of rock, WAT camp, $1.9 \mathrm{~km}$ WNW. of Mt Lindsay, ex grass, $27^{\circ} 01^{\prime} 41^{\prime \prime S}, 129^{\circ} 51^{\prime} 56^{\prime \prime} \mathrm{E}, 16-19$ October 1996, Pitjantjatjara Lands Survey (SAM N1999498); $1 \delta, 6 \mathrm{~km}$ WSW. of Womikata Bore Homeland, pitfall, $26^{\circ} 06^{\prime} 33^{\prime \prime} \mathrm{S}, 1^{\circ} 2^{\circ} 05^{\prime} 57^{\prime \prime} \mathrm{E}, 18-21$ October 1994, J.A. Forrest, Pitjantjatjara Lands Survey (SAM N199588). Victoria: $1 \delta, 10 \mathrm{~km}$ E. of Edenhope, 36 $59^{\circ} \mathrm{S}, 1^{\circ} 1^{\circ} 23^{\prime} \mathrm{E}, 20$ September 1980, DH (SAM N199595). Western Australia: 2 , Ajana Back Road [W. of Ajana], site NO 7, wet pitfall trap, 2759'57"S, 114³7'55"E, 15 September 1998 - 30 March 1999, NG, CALM [Conservation and Land Management Dept] /SAP Survey (WAM T42244, T44819); $1 \delta$, Amery-Benjabbering Road, near Amery (at turnoff to Minnivale), $31^{\circ} 09^{\prime} \mathrm{S}, 117^{\circ} 06^{\prime} \mathrm{E}$, 20 May 1996, MSH (WAM 98/1604); 1 ð, 2 , Askew Road [S. of Mollerin Lake], site BE 6, wet pitfall trap, $30^{\circ} 35^{\prime} 46^{\prime \prime S}, 117^{\circ} 54^{\prime} 28^{\prime \prime E}, 15$ September 1998 - 8 April and 25 October 1999, LK, CALM/ SAP Survey (WAM T42242); 2 , Bendering East Reserve Road, site $\mathrm{KN} \mathrm{10,} \mathrm{wet} \mathrm{pitfall} \mathrm{trap,}$ 32²3'57"S, 118²3'12"E, 30 October - 19 May 1998, PVH, CALM/SAP Survey (WAM T41945); 1 ㅇ, Bendering Reserve Road, site KN 11, wet pitfall trap, 32²1'27'S, 118 $29^{\prime} 46^{\prime \prime} \mathrm{E}, 30$ October 1997 - 19 May 1998, PVH, NG, CALM/SAP Survey (WAM T41946); 1 \%, Boyup Brook - Arthur River, site DA

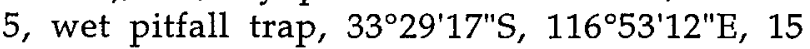
October 1999 - 1 November 2000, PVH et al., CALM/SAP Survey (WAM T44820); 1 o, 1 q, Broom Gully, Di Russo Road, site HY 13, wet pitfall trap, 32 $36^{\prime} 53^{\prime \prime} S, 1^{\circ} 06^{\prime} 10^{\prime \prime} \mathrm{E}, 30$ October 1997 - 19 May 1998, PVH, NG, CALM/SAP Survey (WAM T41931); 6 \%, 1 \%, Bruce Rock - Doodlakine Road, site KL 4, wet pitfall trap, 31 $51^{\prime} 26^{\prime \prime} \mathrm{S}, 118^{\circ} 06^{\prime} 14^{\prime \prime} \mathrm{E}$, 30 October 1997 - 22 May 1998, PVH, NG, CALM/ 
SAP Survey (WAM T41934 - 6); 2 ð, same data except 22 May - 29 September 1998, NG (WAM T44821); 2 s, Bunce - King Road, NE. of Darkan, site DA 3, wet pitfall trap, $33^{\circ} 18^{\prime} 47^{\prime \prime} S, 116^{\circ} 48^{\prime} 22^{\prime \prime} E$, 15 October 1999 - 1 November 2000, PVH et al., CALM/SAP Survey (WAM T44822); 1 \%, Buntine Nature Reserve, West, site WU 2, wet pitfall trap,

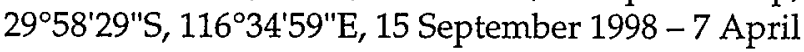
and 25 October 1999, LK, CALM/SAP Survey (WAM T42245); $1 \delta$, Buntine Nature Reserve, North, site WU 3, wet pitfall trap, 2958'16"S, 116 35'05"E, 15 September 1998 - 7 April and 25 October 1999, BD, CALM/SAP Survey (WAM T42246); 1 \& , Buntine Nature Reserve, East, site WU 5 , wet pitfall trap, $29^{\circ} 58^{\prime} 38^{\prime \prime} \mathrm{S}, 116^{\circ} 34^{\prime} 27^{\prime \prime} \mathrm{E}, 15$ September 1998 - 25 October 1999, LK, CALM/SAP Survey (WAM T44823); 1 \%, Burma Road [Nature] Reserve [29 $00^{\prime} S, 115^{\circ} 05^{\prime} E$ ], 18 September 1986, R.P. McMillan (WAM 99/208); 1 \%, Burracoppin Nature Reserve, Powerline, site MN 3, wet pitfall trap, 31'24'39"S, 118³0'33"E, 30 October 1997 - 21 May 1998, PVH, NG, CALM/SAP Survey (WAM T41947); 2 o, same data except 21 May - 22 September 1998, NG (WAM T44824); 1 ㅎ, 5 우, Comitun Dam Nature Reserve, North, site KL 5, wet

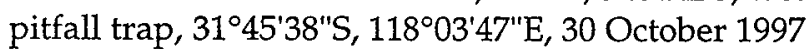
- 22 May 1998, PVH, NG, CALM/SAP Survey (WAM T41937 - 40); 1 oे, Commonwealth Road,

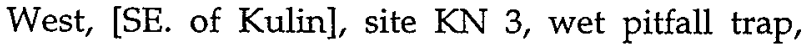
$32^{\circ} 44^{\prime} 13^{\prime \prime} \mathrm{S}, 118^{\circ} 16^{\prime} 16^{\prime \prime} \mathrm{E}, 30$ October 1997 - 15 May 1998, BD, CALM/SAP Survey (WAM T42247); 1 , ,

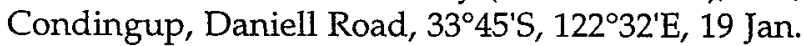
1999, AFL (WAM T40623); 1 \%, Cookinbin Nature Reserve, site MN 8, wet pitfall trap, $31^{\circ} 00^{\prime} 05^{\prime \prime} S$, $118^{\circ} 14^{\prime} 00^{\prime \prime E}, 30$ October - 15 December 1997, PVH, CALM/SAP Survey (WAM T41949); 3 \%, Corackerup Nature Reserve, site 01, pitfall trap, 34²'12'S, 11842'00"E, October 1996, A. Sanders (WAM 98/1605-7); 2 o, Coyrecup Lake Nature Reserve, West, site DU 7, wet pitfall trap, 3343'07"S, $117^{\circ} 51^{\prime} 18^{\prime \prime E}, 15$ October $1999-1$ November 2000, PVH et al., CALM/SAP Survey (WAM T44825); 1 q, Cunderdin Road, North, NE. of Mukinbudin, site BE 4, wet pitfall trap, 30³8'03"S, $118^{\circ} 29^{\prime} 03^{\prime \prime} \mathrm{E}, 15$ September 1998 - 9 April and 25 October 1999, LK, CALM/SAP Survey (WAM T42248); 1 오, Darlington [31⒌'S, $\left.116^{\circ} 05^{\prime} \mathrm{E}\right]$, 1975-1979, G. H. Lowe (WAM T40624); 1 సै, Dryandra Forest, Attunga Road, Skeleton Block, site NR 3, wet pitfall trap, $32^{\circ} 46^{\prime} 58^{\prime \prime S}, 116^{\circ} 51^{\prime} 54^{\prime \prime} \mathrm{E}, 30$ October 1997 - 14 May 1998, NG, CALM/SAP Survey (WAM T42249); 1 $\delta, 1$, Dryandra [Forest], Patonga Road, Smith Block, site NR 4, wet pitfall

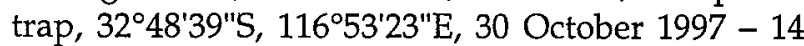
May 1998, E. Ladhams, CALM/SAP Survey (WAM T41953); 2 o, same data except 14 May - 16 September 1998, LK (WAM T44837); 1 ơ, Drysdale River Station, in scrub, $15^{\circ} 42^{\prime} \mathrm{S}, 126^{\circ} 23^{\prime} \mathrm{E}, 30$ May 1993, AFL (S. 1225) (WAM 98/1608); 1 đo, Drysdale
River Station, Woodhouse River Crossing area, [c. $15^{\circ} 42^{\prime} \mathrm{S}, 126^{\circ} 22^{\prime} \mathrm{E}$ ], 5 August 1993, AFL (S. 1320) (WAM 98/1609); $1 \delta$, Drysdale River Station, in truck, $15^{\circ} 42^{\prime} \mathrm{S}, 126^{\circ} 23^{\prime} \mathrm{E}, 10$ December 1993, AFL (S. 1454) (WAM 98/1610); 2 \%, 2 juveniles, Durokoppin [Nature] Reserve, sites H1, H2, wet pitfalls, $31^{\circ} 30^{\prime} \mathrm{S}, 117^{\circ} 44^{\prime} \mathrm{E}, 13-23$ March 1992, GF (WAM 98/2125-8); 2 o, same data except sites H3, J1, 3-13 November 1992 (WAM 98/1611, T40625); 3 q, Durokoppin Nature Reserve, North, site KL 8, wet pitfall trap, $31^{\circ} 24^{\prime} 09^{\prime \prime} \mathrm{S}, 117^{\circ} 45^{\prime} 32^{\prime \prime} \mathrm{E}, 30$ October 1997 - 22 May 1998, PVH, NG, CALM/SAP Survey (WAM T41941 - 2); 1 б, Durokoppin Nature Reserve, South, site KL 10, wet pitfall trap, $31^{\circ} 24^{\prime} 46^{\prime \prime} \mathrm{S}, 117^{\circ} 45^{\prime} 16^{\prime \prime} \mathrm{E}, 30$ October 1997 - 22 May 1998, PVH, NG, CALM/SAP Survey (WAM T41943); 1 б, 1 q, East Yorkrakine [Nature] Reserve, sites K4, J2, [31 $\left.28^{\circ} \mathrm{S}, 117^{\circ} 41^{\prime} \mathrm{E}\right], 3-14$ November 1988, D. Mitchell et al. (WAM 98/1612, T42051)); $4 \delta$, same data except sites J2, J4, K3, 1929 May 1989, GF (WAM 98/1613-6); 1 $\delta$, same data except site K1, 3-13 November 1989 (WAM 98/ 1617); $1 \delta$, same data except site J4, 17-27 November 1989 (WAM T44323); 1 $\approx$, same data except site K3, 18-28 March? 1990 (WAM T44322); $2 \sigma$, Elashgin Nature Reserve, granite site, wet pitfall trap, $31^{\circ} 20^{\prime} 07^{\prime \prime} \mathrm{S}, 117^{\circ} 26^{\prime} 10^{\prime \prime} \mathrm{E}, 7$ September 29 November 1999, JMW, I.Studley (WAM T40633); 1 t, Eneabba, R.G.C. Mineral Sands, site 1, pitfall trap, $2^{\circ} 56^{\prime} S, 115^{\circ} 17^{\prime} \mathrm{E}, 30$ October 1997, R.P. McMillan (WAM 98/1941); 1 $\star$, same data except site 3, 31 October 1997 (WAM T40626); 1 \&, Erikin Road, site KL 2, wet pitfall trap, 31 $57^{\prime} 51^{\prime \prime S}$, 11756'05"E, 30 October 1997 - 22 May 1998, PVH, NG, CALM/SAP Survey (WAM T41932); 2 ¿ , Gidgegannup, Waterford Road, [31 ${ }^{\circ} 48^{\prime} \mathrm{S}, 116^{\circ} 11^{\prime} \mathrm{E}$ ], 12 January 1992, JMW (WAM 98/1618-9); 1 \%, Glen Helen Gorge, $25^{\circ} 00^{\prime} S, 128^{\circ} 09^{\prime} \mathrm{E}, 14$ January 1990 , MSH, T.F. Houston (WAM 98/1620); 1 ð, 1 ㅇ, Gura Road, East, [N. of Congelin], site NR 9, wet pitfall trap, 32०46'09"S, $116^{\circ} 57^{\circ} 08^{\prime \prime} \mathrm{E}, 30$ October $1997-12$ May 1998, NG, CALM/SAP Survey (WAM T42250); $1 \delta$, Heitmans Scrub, site M4, pitfalls, $31^{\circ} 31^{\prime} S$, 117³7'E, 16-26 March 1989, GF et al. (WAM 98/ 1621); 1 đ, same data except 3-13 November 1992 (WAM 98/1622); 1 \%, Hotham River National Park, site NR 1, wet pitfall trap, $32^{\circ} 36^{\prime} 40^{\prime \prime} S, 117^{\circ} 06^{\prime} 00^{\prime \prime} \mathrm{E}$, 15 May - 16 September 1998, PVH, CALM/SAP Survey (WAM T44826); 2 q, Howatharra Nature Reserve, site NO 3, wet pitfall trap, $28^{\circ} 32^{\prime} 45^{\prime \prime} S$, 114³9'56"E, 15 September 1998 - 18 October 1999, NG, CALM/SAP Survey (WAM T42251); 1 б, 20 $\mathrm{km}$ E. of Hyden, [c. $32^{\circ} 25^{\prime} \mathrm{S}, 119^{\circ} 02^{\prime} \mathrm{E}$ ], August 1990, D. Knowles (WAM 98/1623); 1 đ, NE. of Jarrahdale, Alcoa minesite, site 1 (NE. of Johnson/ Chandler Rds Junction), [c. 32 $17^{\prime} \mathrm{S}, 116^{\circ} 06^{\prime} \mathrm{E}$ ], 1986, P.R. Mawson (WAM T44708); $1 \delta^{\circ}$, Jilakin Lake, site $\mathrm{KN} 1$, wet pitfall trap, $32^{\circ} 40^{\prime} 29^{\prime \prime} \mathrm{S}, 118^{\circ} 20^{\prime} 10^{\prime \prime} \mathrm{E}, 30$ October 1997 - 15 May 1998, PVH, NG, CALM/ 
SAP Survey (WAM T41944); $1 \delta$, same data except LK (WAM T42252); ); 10 , Jingemia Hill, Watheroo National Park, site DN 2, wet pitfall trap, $30^{\circ} 15^{\prime} 21^{\prime \prime S}, 116^{\circ} 01^{\prime} 17^{\prime \prime} \mathrm{E}, 15$ October $1999-1$ November 2000, PVH et al., CALM/SAP Survey (WAM T44827); 3 \% , Jouerdine Nature Reserve, site BE 3, wet pitfall trap, $30^{\circ} 38^{\prime} 11^{\prime \prime} S, 118^{\circ} 25^{\prime} 39^{\prime \prime} \mathrm{E}, 15$ September 1998 - 9 April and 25 October 1999, LK, CALM/SAP Survey (WAM T42253, T44828); 2 ㅇ, Julimar Conservation Park, NE., site JB 8, wet pitfall trap, 3123'59"S, 116²19'06"E, 15 September 1998 22 April and 4 November 1999, BD, CALM/SAP Survey (WAM T44829); 1 s, Julimar Conservation Park, North, site JB 12, wet pitfall trap, 3124'06"S, 11609'49"E, 15 September 1998 - 4 November 1999, NG, CALM/SAP Survey (WAM T44830); 3 o', same data except site JB 13, 3121'21"S, 116 13'04"E, BD (WAM T42254); 2 \%, SE. of Kadji Kadji, North along powerline, site $\mathrm{MO} 8$, wet pitfall trap, $29^{\circ} 10^{\prime} 18^{\prime \prime} \mathrm{S}$, $116^{\circ} 23^{\prime} 16^{\prime \prime E}, 15$ September 1998 - 1 April 1999, BD, CALM/SAP Survey (WAM T42255); 1 đ , Karlgarin National Park, Hyden Road, site KN 5, wet pitfall trap, 32²9'59'S, 118 33'05"E, 15 October 1997 - 19 May 1998, PVH, CALM/SAP Survey (WAM $\mathrm{T} 42256) ; 1 \delta^{\circ}, 1$ ㅇ, Kellerberrin, 31 $38^{\prime} \mathrm{S}, 117^{\circ} 43^{\prime} \mathrm{E}$, no date, G.T. Smith (WAM 98/1525-6); 1 , Koorda Road, nature reserve \#22363, site WH 6, wet pitfall trap, $30^{\circ} 45^{\prime} 25^{\prime \prime} \mathrm{S}, 117^{\circ} 05^{\prime} 26^{\prime \prime} \mathrm{E}, 15$ September 1998 29 April and 25 October 1999, BD, CALM/SAP Survey (WAM T44831); 1 \%, Kulunilup Nature Reserve, East, site UN 8, wet pitfall trap, $34^{\circ} 20^{\prime} 00^{\prime \prime} S$, $116^{\circ} 48^{\prime} 03^{\prime \prime E}, 15$ October 1999 - 1 November 2000, NG, CALM/SAP Survey (WAM T44832); 1 ㅇ, Lake Campion Nature Reserve, site MN 12, wet pitfall

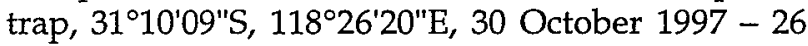
April 1998, E. Ladhams, CALM/SAP Survey (WAM T41952); 2 б, 1 ㅇ, Lake Fox, site HY 9, wet pitfall trap, 32 ${ }^{\circ} 54^{\prime} 50^{\prime \prime} \mathrm{S}, 119^{\circ} 28^{\prime} 32^{\prime \prime} \mathrm{E}, 30$ October 1997 - 20 May 1998, PVH, CALM/SAP Survey (WAM T41930); 2 \%, same data except 20 May - 29 August 1998 (WAM T42257); 1 б, 3 q, Lake Gulson, site HY 8, wet pitfall trap, $32^{\circ} 47^{\prime} 11^{\prime \prime} \mathrm{S}, 119^{\circ} 22^{\prime} 07^{\prime \prime} \mathrm{E}, 30$ October 1997 - 20 May 1998, E. Ladhams, CALM/ SAP Survey (WAM T41929); 1 đ, 1 \%, Lily McCarthy Rock, site HY 10, wet pitfall trap, $32^{\circ} 41^{\prime} 35^{\prime \prime}$, $119^{\circ} 20^{\prime} 25^{\prime \prime E}, 30$ October 1997 - 20 May 1998, E. Ladhams, CALM/SAP Survey (WAM T42259); 2 \&, Lochada Road Nature Reserve, West, site MO 9, wet pitfall trap, $2^{\circ} 15^{\prime} 13^{\prime \prime} S, 116^{\circ} 22^{\prime} 05^{\prime \prime} \mathrm{E}$, 15 September 1998 - 1 April 1999, LK, CALM/SAP Survey (WAM T44834); 1 đ, Mackie Creek Reserve, East, site $Y O 1$, wet pitfall trap, 31 $59^{\prime} 33^{\prime \prime} S$, $117^{\circ} 01^{\prime} 19^{\prime \prime E}, 20 \mathrm{May}$ - 5 November 1998, BD, CALM/SAP Survey (WAM T44835); 1 t, Manmanning Dam Nature Reserve, SE., site WH 8, wet pitfall trap, $30^{\circ} 54^{\prime} 53^{\prime \prime} \mathrm{S}, 117^{\circ} 05^{\prime} 41^{\prime \prime} \mathrm{E}, 15$ September $1998-25$ and 28 October 1999, LK, CALM/SAP Survey (WAM T42260); 1 으, Manmanning Dam Nature Reserve, site WH 7, wet

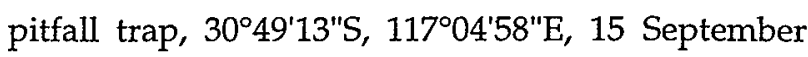
1998 - 29 April and 25 October 1999, LK, CALM/ SAP Survey (WAM T42261); 1 ㅇ, Mollerin Lake, South, site BE 8, wet pitfall trap, 30'31'47"S, $117^{\circ} 34^{\prime} 45^{\prime \prime} \mathrm{E}, 15$ September 1998 - 25 October 1999, LK, CALM/SAP Survey (WAM T44833); 1 , Mollerin Lake, West, site BE 10, wet pitfall trap, 30³1'41"S, 117³3'54"E, 15 September 1998 - 8 April and 25 October 1999, PVH, CALM/SAP Survey (WAM T42258); 2 o, [S. of Mollerin Lake], Rock, Long Muir Road, West, site BE 7, wet pitfall trap, 30³2'50"S, 117³3'56"E, 16 September 1998 - 8 April and 25 October 1999, PVH, CALM/SAP Survey (WAM T42243); 4 o , 3 \&, Moonijin Nature Reserve, site WH 11, wet pitfall trap, 3054'57"S, $117^{\circ} 09^{\prime} 05^{\prime \prime} \mathrm{E}$, 15 September 1998 - 25 October 1999, BD, CALM/ SAP (WAM T42262, T 44836); 2 \%, Mount Moore Nature Reserve, site $\mathrm{MN} \mathrm{7,} \mathrm{wet} \mathrm{pitfall} \mathrm{trap,}$ 31'12'45"S, 118¹8'15"E, 30 October - 15 December 1997, PVH, CALM/SAP Survey (WAM T41948); 2 ๙ 1 1 , same data except 15 October 1997-21 May 1998 (WAM T42264); 1 , , Mount Stirling Road, site QU 10, wet pitfall trap, 31'59'27"S, 117²4'19"E, 30 October 1997 - 27 May 1998, PVH, NG, CALM/ SAP Survey (WAM T41954); $3 \delta, 5 \%$, Noorajin Soak Nature Reserve, site WH 13, wet pitfall trap, 3045'17"S, $117^{\circ} 14^{\prime} 30^{\prime \prime} \mathrm{E}, 15$ September 1998 - 18 October 1999, NG, CALM/SAP Survey (WAM T42263); 2 \%, Packsaddle Plain via Kununurra, $15^{\circ} 49^{\prime} \mathrm{S}, 128^{\circ} 43^{\prime} \mathrm{E}, 26$ October 1999, A. Postle (WAM T41824); 1 ㅇ, Parmelia, 15 Sicklemore Road, $32^{\circ} 15^{\prime} \mathrm{S}$, $115^{\circ} 49^{\prime} \mathrm{E}, 25$ December 1999, A.E. de Jong (WAM T42160); 1 \%, Quairading Railway Water Supply, West, site QU 11, wet pitfall trap, $32^{\circ} 01^{\prime} 32^{\prime \prime S}$, 117²1'58"E, 20 May - 5 October 1998, BD, CALM/ SAP Survey (WAM T44838); 1 ơ, 1 \&, same data except 30 October - 27 May 1998, E. Ladhams (WAM T42265); 1 $\%$, same data except West, site QU 12, 32 $01^{\prime} 15^{\prime \prime} S, 117^{\circ} 22^{\prime} 57^{\prime \prime} \mathrm{E}, \mathrm{PVH}, \mathrm{NG}$ (WAM T41956); 1 \%, 1 \&, St Ronans Nature Reserve, North, site YO 12, wet pitfall trap, 31 $52^{\prime} 30^{\prime \prime} \mathrm{S}, 116^{\circ} 36^{\prime} 10^{\prime \prime} \mathrm{E}$, 30 October 1997 - 26 May 1998, E. Ladhams, CALM/SAP Survey (WAM T42266); 2 \%, Salt River, Beverley Road, site QU 8, wet pitfall trap, $32^{\circ} 05^{\prime} 02^{\prime \prime} \mathrm{S}, 117^{\circ} 23^{\prime} 33^{\prime \prime} \mathrm{E}, 30$ October 1997 - 26 May 1998, PVH, NG, CALM/SAP Survey (WAM T41955); 10 , Sandplains Nature Reserve, West, site MN 4, wet pitfall trap, $31^{\circ} 35^{\prime} 48^{\prime \prime} \mathrm{S}, 118^{\circ} 45^{\prime} 24^{\prime \prime} \mathrm{E}, 28$ April - 22 September 1998, NG, CALM/SAP

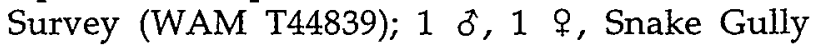
Nature Reserve, site WU 11, wet pitfall trap, 30¹3'05"S, 116 56'36"E, 15 September 1998 - 7 April 1999, NG, CALM/SAP Survey (WAM T42267); $1 \delta$, Talgomine Reserve, North, site MN 10, wet pitfall trap, $31^{\circ} 14^{\prime} 40^{\prime \prime} \mathrm{S}, 118^{\circ} 24^{\prime} 25^{\prime \prime} \mathrm{E}, 30$ October - 15 December 1997, PVH, CALM/SAP Survey (WAM T41950); 1 \& , same data except 10 October 1997- 28 April 1998, PVH, NG (WAM T41951); 1 б, Tutanning Nature Reserve, East, site WK 8, wet 


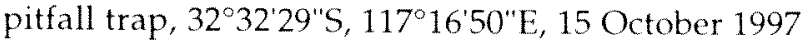
- 11 May 1998, PVH, CALM/SAP Survey (WAM T42268); 4 9, 2 juvs, Uwara Nature Reserve, North, site ML 7, wet pitfall trap, $28^{\circ} 24^{\prime} 02^{\prime \prime} S, 115^{\circ} 34^{\prime} 34^{\prime \prime} \mathrm{E}$, 15 September 1998 - 1 April and 18 October 1999, BD, CALM/SAP Survey (WAM T44840); 1 \%, 1 , Walkaway Nature Reserve, site WH 12, wet pitfall

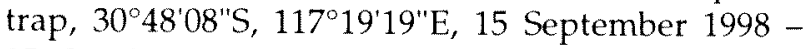
25 October 1999, BD, CALM/SAP Survey (WAM T44841); 1 ठ , 1 \%, Wansbrough Nature Reserve, North, site ST 11, wet pitfall trap, 34 $09^{\circ} 08^{\prime \prime} \mathrm{S}$, $117^{\circ} 40^{\prime} 09^{\prime \prime} \mathrm{E}, 18$ October 1999 - 30 May 2000, NG, CALM/SAP Survey (WAM T44842); $1 \delta^{*}$, private property E. of Watheroo National Park, site DN 4, wet pitfall trap, $30^{\circ} 09^{\prime} 17^{\prime \prime} \mathrm{S}, 115^{\circ} 57^{\prime} 38^{\prime \prime} \mathrm{E}, 15$ October 1999 - 1 November 2000, PVH et al., CALM/SAP Survey (WAM T44843); 2 o, Yarding Nature Reserve, site KL 3, wet pitfall trap, 31055'02"S, 117 58'49"E, 30 October 1997 - 22 May 1998, PVH, NG, CALM/SAP Survey (WAM T41933); 3 \%, same data except 22 May - 29 September 1998, LK (WAM T44844); 1 \&, Yelbeni, W. edge, wet pitfall trap, $31^{\circ} 10^{\prime} 24^{\prime \prime S}, 117^{\circ} 39^{\prime} 32^{\prime \prime E}, 7$ September - 30 November 1999, JMW, I. Studley (WAM T42052); 2 ¿, Yorkrakine Rock Nature Reserve, site at base of rock, wet pitall trap, $31^{\circ} 25^{\prime} 05^{\prime \prime} \mathrm{S}, 117^{\circ} 30^{\prime} 56^{\prime \prime} \mathrm{E}, 6$ September - 29 November 1999, JMW, I. Studley (WAM T40634).

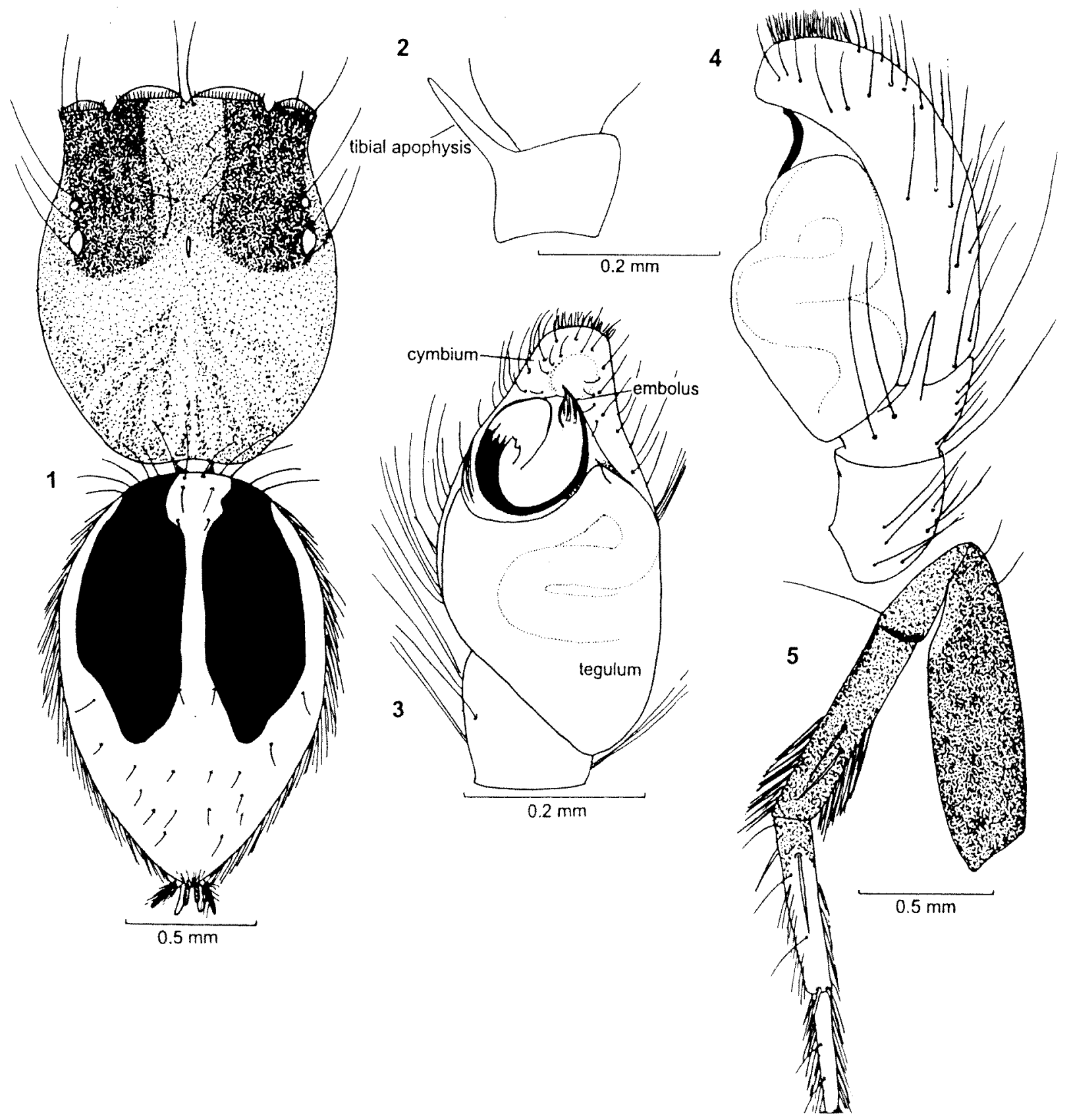

Figures 1-5 Lycidas chrysomelas (Simon): 1, cephalothorax and abdomen, dorsal; 2, left pedipalp, tibial apophysis, dorsal; 3, left pedipalp, ventral; 4, left pedipalp, retrolateral; 5, right leg III, retrolateral. 0 . WAM 98/1525. 


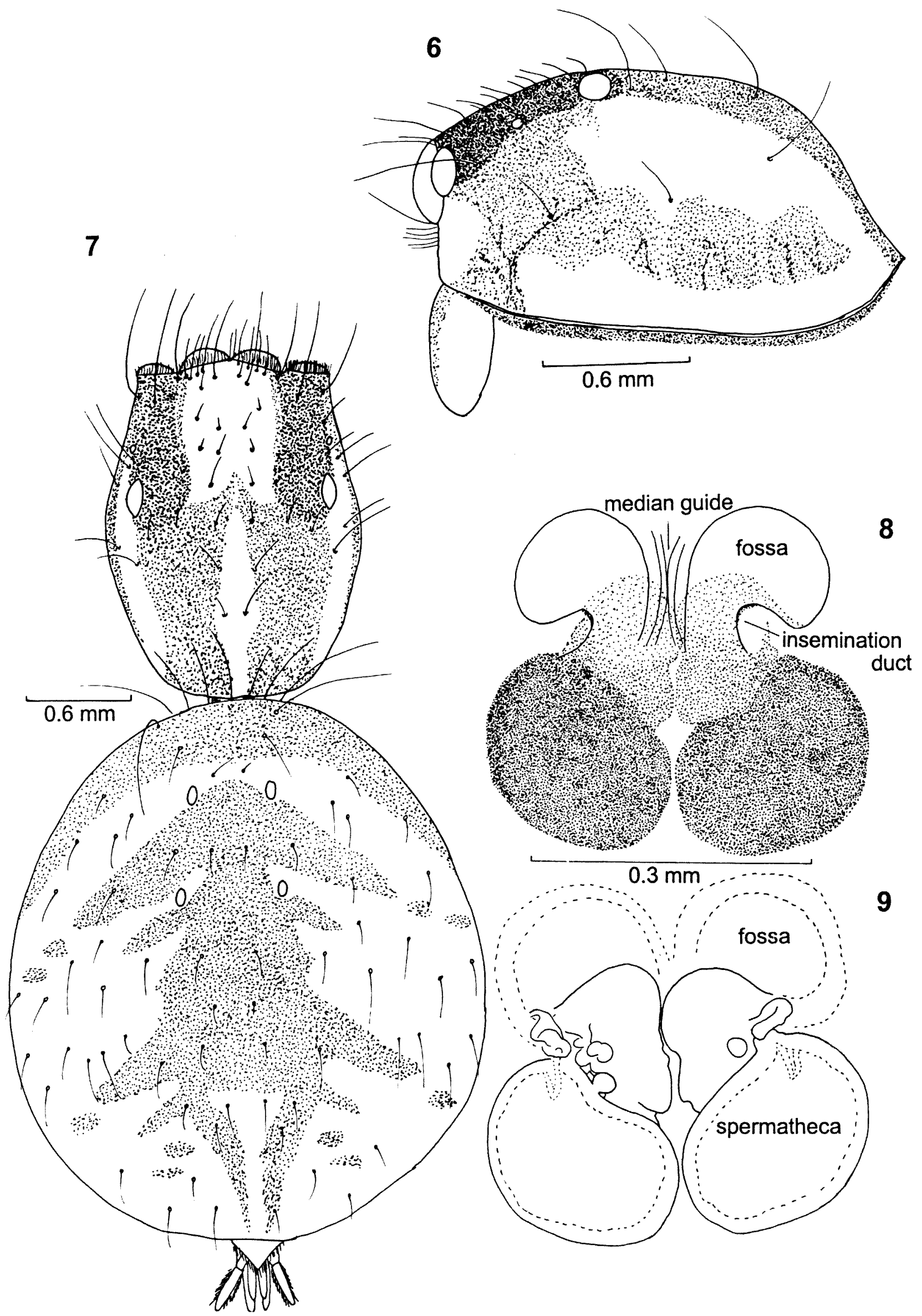

Figures 6-9 Lycidas chrysomelas (Simon): 6, cephalothorax, lateral; 7, cephalothorax and abdomen, dorsal; 8, epigyne, ventral; 9, epigyne, dorsal. ?, WAM 98/1526. 


\section{Diagnosis}

Lycidas chrysomelas differs from all other Lycidas species, both described and undescribed, by the following combination of characters: male abdomen with metallic dark green/peacock blue dorsal patch with 2 ovoid black sections; male tibia III with brush of black hairs; male metatarsus III and tarsus III with brush of white hairs; males and females with two dark brown to black broad stripes extending from anterior lateral eyes to posterior of posterior lateral eyes; female with greyish border around edge of cephalothorax and with broad, short copulatory canals and reniform spermathecae.

\section{Description}

\section{Male (WAM 98/1525)}

Cephalothorax black to dark brown with lighter brown bands laterally; cephalic area with short black hairs on dark brown extending from anterior lateral eyes to edge of cephalic area; thoracic area dark brown with scattered white hairs and central strip of white hairs extending to posterior. Clypeus brown, chelicerae brown to tan distally. Maxillae, labium tan with cream edges. Anterior eyes fringed with creamy white hairs along dorsal edge. Abdomen oval in shape; dorsum of abdomen with dark green to peacock blue metallic pattern of short squamous hairs and two elongate patches of black which extend over anterior two-thirds of abdomen; brown and ginger bristles present along edges of abdomen, longer on side than posterior (Figure 1). Sternum dark brown; venter of abdomen tan with scattered greyish patches; spinnerets $\tan$ to cream with dark grey patches. Pedipalp light tan with grey patches; tibial apophysis with broad base, straight (Figure 2); proximal two-thirds of cymbium, dorsal tibia and dorsal patella of pedipalp densely covered with medium-length white hairs, distal third of cymbium with medium-length brown hairs; embolus coiled loosely, falcate, with single broad, twisted tip; tegulum not developed into posterior lobe (Figures 3,4). Legs: femora, patella of legs I, II, III brown, femora IV, pale brown, patella IV yellow; remainder of legs I, II, IV orange with dark areas at joints; tibia III brown with brush of black hairs on dorsal and ventral sides; metatarsus III and tarsus III with brush of white hairs on dorsal and ventral sides; tarsus III brush denser dorsally and extends over tips of claws (Figure 5).

\section{Female (WAM 98/1526)}

Cephalothorax dark brown with pale yellow bands laterally and with greyish border around edge (Figure 6); cephalic area with short black hairs on dark brown extending from anterior lateral eyes to edge of cephalic area; thoracic area dark brown with scattered white hairs and central strip of white hairs extending to posterior. Anterior eyes fringed with creamy white hairs along dorsal edge. Abdomen yellow, to creamy white, dorsum and venter speckled with greyish markings (Figure 7). Clypeus, chelicerae tan; maxillae, labium, sternum pale yellow. Spinnerets cream with grey patches. Epigyne lacking proximal receivers, fossae large, oval-shaped, accessory glands present; spermathecae large, kidney-shaped with translucent tubules overlying broad, short copulatory canals. Insemination duct openings midway along posterior edge of fossae (Figures 8, 9). Legs: femora I-IV pale yellow, remainder of all legs light brown. All leg segments with patches of grey, especially at joints.

Dimensions ( $\mathrm{mm}$ ). \%, WAM 98/1525 ( 9 , WAM 98/ 1526): total length (excluding chelicerae) 3.63 (5.00). Carapace length 1.50 (1.81). Abdomen length 1.75 (3.12). Leg I: femur $0.69(0.75)$, patella $0.44(0.63)$, tibia $0.44(0.44)$, metatarsus $0.37(0.44)$, tarsus 0.37 (0.37). Leg II: femur $0.69(0.75)$, patella $0.44(0.56)$, tibia $0.44(0.50)$, metatarsus $0.37(0.44)$, tarsus 0.37 (0.37). Leg III: femur 1.44 (1.44), patella $0.63(0.63)$, tibia $1.00(0.94)$, metatarsus $0.63(0.81)$, tarsus 0.44 (0.63). Leg IV: femur 0.94 (1.13), patella $0.44(0.56)$, tibia $0.63(0.75)$, metatarsus $0.63(0.94)$, tarsus 0.50 (0.63). Legs, relative lengths: III:IV:I=II (III:IV:I:II).

\section{Remarks}

Lycidas chrysomelas occurs in semi-arid regions of mainland Australia. It ranges from semi-tropical north-western Western Australia (Drysdale River Station) to mediterranean southern Australia, and east to Finley, New South Wales (Figure 10). However, there are large gaps in the known distribution and no specimens have been recorded from the Northern Territory or Queensland but this may be due to a collection artefact. Over the past 10-15 years there has been an increase in surveying of the semi-arid areas of Australia with more emphasis being placed on the collection of invertebrates. A small spider such as L. chrysomelas can be easily overlooked and the majority of additional material of this species has been collected in pitfall traps. The lack of specimens from the Northern Territory, inland Queensland and central Western Australia may be due to lack of appropriate collecting and this species may yet be found in suitable parts of these areas. Collection data indicate that $L$. chrysomelas can be active at all months of the year except June and July.

Based on male pedipalpal features $L$. chrysomelas appears to be closely related to L. nigromaculatus (Keyserling) (Żabka, 1987) and L. auripes (L. Koch). Also L. chrysomelas female genitalia shows many features in common with L. bitaeniatus (Keyserling).

\section{ACKNOWLEDGEMENTS}

I thank the following people for the loan of 


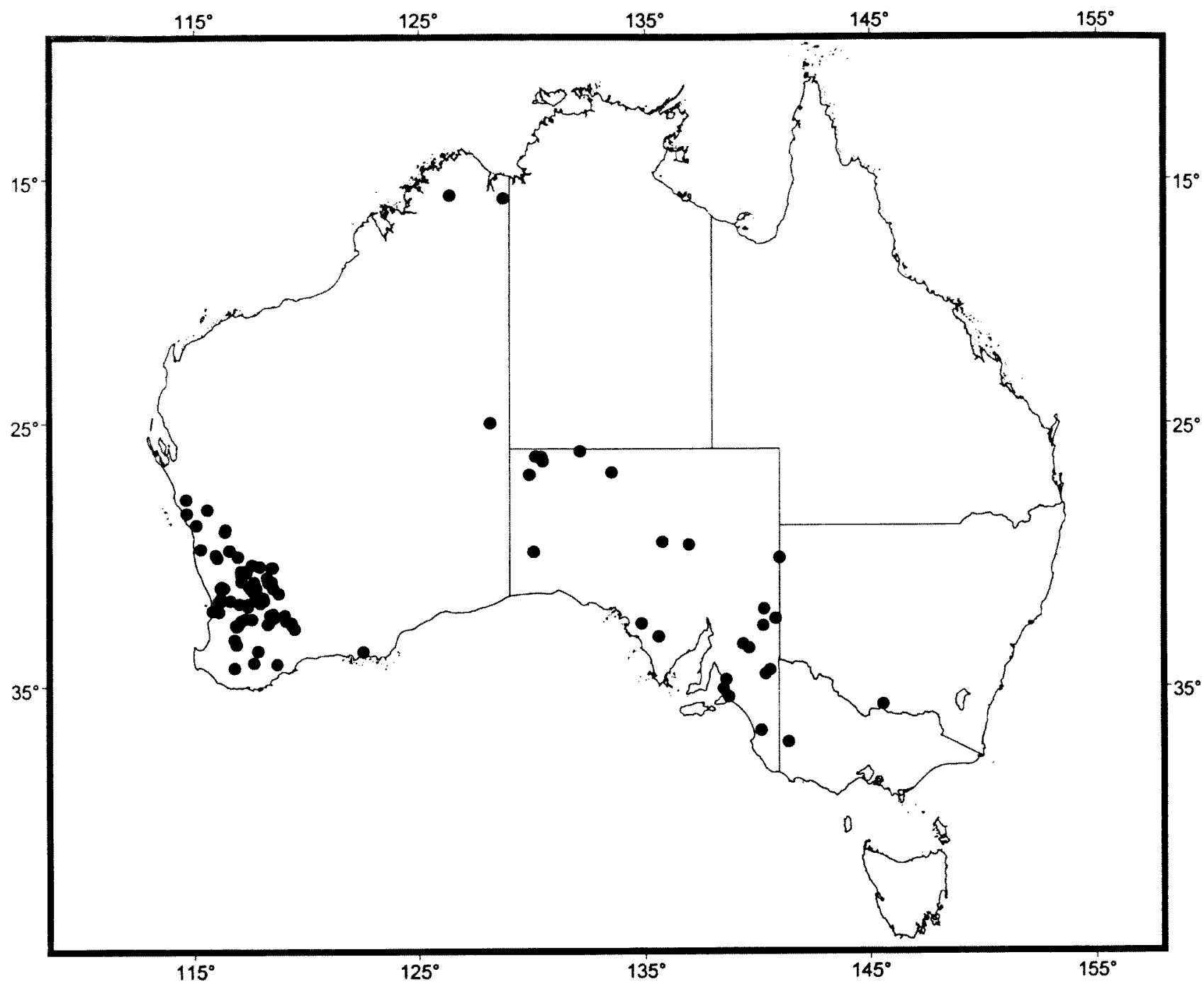

Figure 10 Map of Australia with the collection localities of Lycidas chrysomelas (Simon) indicated by solid circles.

material in their care: Mr D. Hirst (SAM), Dr S. Nawai and Dr J. Dunlop (ZMB), Dr A. Yen, Ms C. McPhee and Mr G. Milledge (NMV). In addition, the team involved in the collection and sorting of the spiders from the Salinity Action Plan Survey under the Conservation and Land Management Dept, W.A., in particular Mr N. McKenzie, Ms N. Guthrie and Mr P. Van Heurck. Also many thanks to Mark Harvey who encouraged me to present this description as a poster at the XIV International Congress of Arachnology, Chicago, U.S.A., 27 June - 3 July 1998 and offered valuable comments on a draft of the manuscript.

\section{REFERENCES}

Bonnet, P. (1957). Bibliographia Araneorum. 2(3). Douladoure, Toulouse.

Karsch, R. (1878). Diagnoses Attoidarum aliquot novarum Novae Hollandiae collectionis Musei zoologici Berolinensis. Mitteilungen des Münchener Entomologischen Verein, München 2: 22-32.

Koch, L. (1881). Die Arachniden Australiens. Vol. 1, pp. 1243-1260. Bauer \& Raspe, Nürnberg.

Rainbow, W. J. (1911). A census of Australian Araneidae. Records of the Australian Museum 9: 107-319.

Roewer, C. F. (1954). Katalog der Araneae. vol. 2. Institut Royal des Sciences Naturelles de Belgique, Bremen.

Simon, E. (1901). Histoire Naturelle des Araignées. vol. 2. Roret, Paris.

Simon, E. (1909). Araneae. In: Die Fauna SudwestAustraliens. vol. 2: 155-122. Gustav Fischer, Jena.

Zabka, M. (1987). Salticidae (Araneae) of Oriental, Australian and Pacific Regions, II. Genera Lycidas and Maratus. Annales zoologici 40: 451-482.

Manuscript received 12 December 2001; accepted 20 May 2002 